\title{
Open
}

\section{TSLP promotes influenza-specific CD8 + T-cell responses by augmenting local inflammatory dendritic cell function}

\author{
K Yadava $^{1}$, A Sichelstiel ${ }^{1}$, IF Luescher ${ }^{2}$, LP Nicod ${ }^{1}$, NL Harris $^{3}$ and BJ Marsland ${ }^{1}$
}

Thymic stromal lymphopoietin (TSLP) is a mucosal tissue-associated cytokine that has been widely studied in the context of T helper type 2 (Th2)-driven inflammatory disorders. Although TSLP is also produced upon viral infection in vitro, the role of TSLP in antiviral immunity is unknown. In this study we report a novel role for TSLP in promoting viral clearance and virus-specific CD8 + T-cell responses during influenza A infection. Comparing the immune responses of wild-type and TSLP receptor (TSLPR)-deficient mice, we show that TSLP was required for the expansion and activation of virus-specific effector CD8 + T cells in the lung, but not the lymph node. The mechanism involved TSLPR signaling on newly recruited CD11b + inflammatory dendritic cells (DCs) that acted to enhance interleukin-15 production and expression of the costimulatory molecule CD70. Taken together, these data highlight the pleiotropic activities of TSLP and provide evidence for its beneficial role in antiviral immunity.

\section{INTRODUCTION}

Influenza virus primarily infects epithelial cells of the upper respiratory tract, leading to cell death and acute inflammation. The immunological mechanisms controlling the protective response against the virus have been the focus of intense research. Indeed, specific roles in the protective response have already been attributed to B cells, distinct dendritic cell (DC) populations, CD4+ T cells, and cytotoxic CD8 + T cells. ${ }^{1-4}$ Upon infection, lungresident DCs migrate to the draining lymphoid tissue and initiate an influenza-specific response through presentation of viral antigens to T cells. Additionally, DCs present within the lungs are necessary for the activation of effector and memory $\mathrm{T}$ cells and the consequent clearance of the virus. ${ }^{5-8}$

The airway epithelium is the primary site of influenza virus infection and replication. Although epithelial cells are known to produce a wide spectrum of inflammatory mediators, the significance of their contribution to anti-influenza responses is poorly understood. Thymic stromal lymphopoietin (TSLP) is an interleukin-7 (IL-7)-like cytokine, which is predominantly produced by epithelial cells. ${ }^{9}$ Several cell types including DCs and CD $4+$ and CD8 + T cells respond to TSLP. To date, TSLP has been studied primarily in the context of T helper type 2 (Th2) responses associated with inflammatory disorders such as atopic dermatitis and asthma ${ }^{10-12}$ and protective immunity to helminth parasites. ${ }^{13,14}$ TSLP can induce Th2 responses either by directly targeting naive CD $4+\mathrm{T}$ cells or indirectly through regulation of DC function. ${ }^{11,15}$ Furthermore, TSLP has been reported to support the survival of cytotoxic T cells both directly ${ }^{16}$ and indirectly through the activation of DCs. ${ }^{17,18}$ Interestingly, viral nucleic acid analogs and proinflammatory cytokines associated with active viral infections are potent inducers of TSLP; ${ }^{19-21}$ however, it has yet to be shown whether TSLP plays a functional role during antiviral immune responses.

To elucidate the role of TSLP in viral infections, we investigated the immune response against influenza $\mathrm{A}$ in mice deficient in TSLP receptor (TSLPR). We found that TSLP expression was induced at day 5 after infection, suggesting that TSLP may not regulate the early innate response to infection but rather adaptive immunity. Indeed, the early kinetics of viral propagation were unaffected by the absence of TSLPR signaling. However, TSLP did play a role in viral clearance at later time points, and the virus-specific CD8 + T-cell response was reduced in the absence of TSLPR.

This defect was not linked to DC migration or priming of virus-specific CD8 + T cells in the draining lymph node; instead,

\footnotetext{
${ }^{1}$ Faculty of Biology and Medicine, University of Lausanne, Service de Pneumologie, Lausanne, Switzerland. ${ }^{2}$ Ludwig Institute for Cancer Research, Epalinges, Switzerland. ${ }^{3}$ Global Health Institute, EPFL-SV-GHI Station 19, EPFL, Lausanne, Switzerland. Correspondence: BJ Marsland (benjamin.marsland@chuv.ch) 
virus-specific CD8 + T-cell responses in the lung were impaired. We found that TSLP targeted CD11b + inflammatory DCs that are recruited to the lungs upon infection and boost local CD8 + T-cell responses. Specifically, TSLP was required to enhance IL-15 production and expression of CD70 by these DCs in the lung. We propose that TSLP enhances cytotoxic T-cell function in the lung indirectly by affecting the maturation of DCs, which are recruited to the lungs upon infection.

\section{RESULTS}

\section{TSLP augments influenza-A-specific CD8 + T-cell responses}

To investigate the role of TSLP in protective antiviral immunity, we first assessed the expression of TSLP in the lung and airways at several time points during infection (Figure 1a).
We found that TSLP expression peaked at day 5 after infection, which coincided with the maximal pulmonary viral load (Figure 1b). We then proceeded to characterize the response against influenza A infection in wild-type C57BL/6 and TSLP receptor (TSLPR)-deficient mice. Analysis of viral load kinetics by both quantitative PCR and tissue culture infectious dose 50 (TCID 50) showed that although early control of viral replication was comparable between wild-type and TSLPR-deficient mice, the absence of TSLP resulted in delayed clearance of the virus from day 7 after infection (Figure 1b). This was not associated with a global reduction in CD8 + T cells in the airways (Figure 1c); however, there was a significant decrease in the number of influenza-specific CD8 + T cells (Figure 1d). In line with this finding, we observed decreased levels of interferon $-\gamma$ production by CD $8+\mathrm{T}$ cells upon specific a

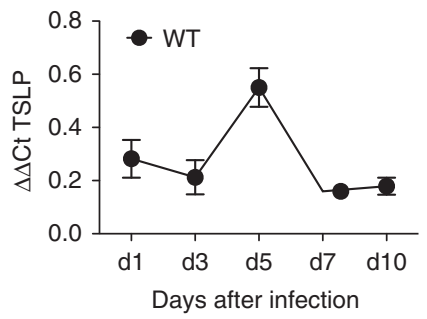

C
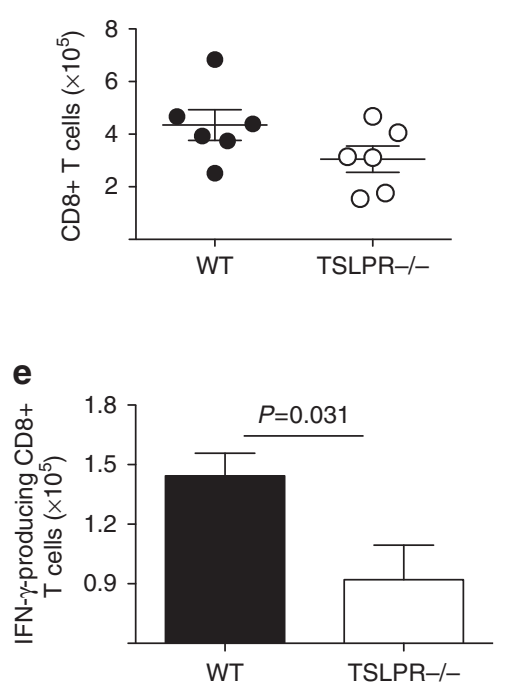

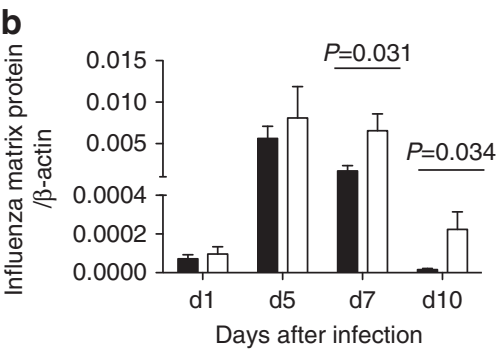

d
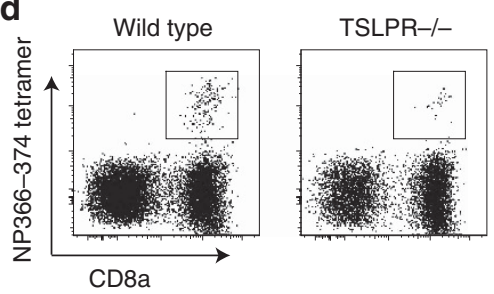

f

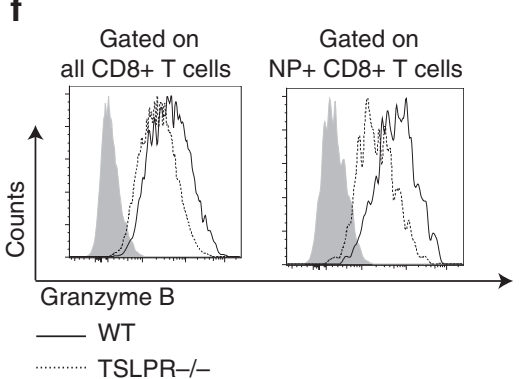

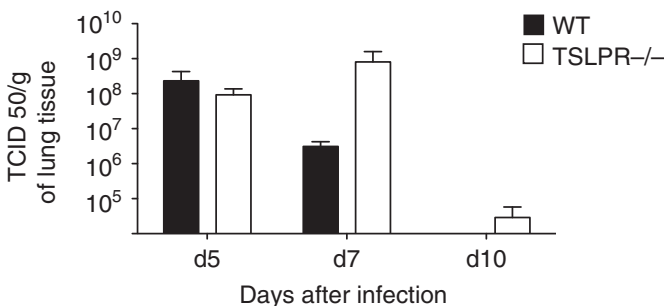
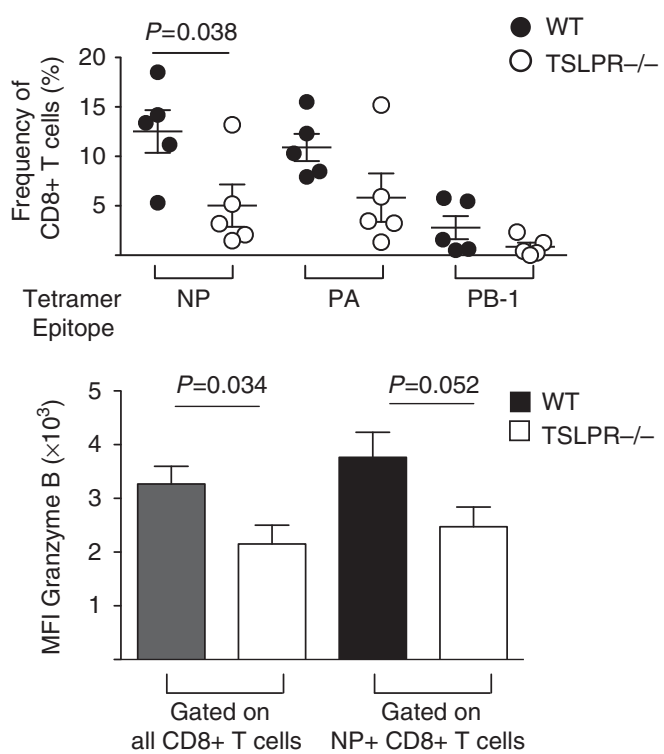

Figure 1 Thymic stromal lymphopoietin (TSLP) is induced during influenza A infection and augments virus-specific CD8+ T-cell responses. Wild-type (WT) and TSLP receptor (TSLPR)-deficient mice were infected intranasally with 50PFU PR8 influenza A virus. (a) TSLP expression was determined by real-time PCR at the indicated time points after infection. (b) Viral load from whole lung and trachea was determined by quantitative real-time PCR. Expression of influenza matrix protein was normalized to $\beta$-actin expression at the indicated time points after infection. The tissue culture infectious dose 50 (TCID 50) was determined on lung homogenate samples at the indicated time points. Data are pooled from two experiments $(n=3-5)$ and are representative of at least four independent experiments. (c) Recruitment of total CD8+ T cells and (d) influenza-specific NP 366-374, PA, and PB-1 CD8 + T tetramer + cells in the bronchoalveolar lavage (BAL) was assessed by flow cytometry on day 10 after infection $(n=5)$. Data are representative of at least three independent experiments. (e) BAL cells were restimulated in vitro by bone marrow-derived dendritic cells (BMDCs) pulsed with ultraviolet (UV)-inactivated virus for $6 \mathrm{~h}$ before analysis of interferon- $\gamma(\mathrm{IFN}-\gamma)$ production. Data are representative of two independent experiments $(n=5-6)$. (f) Granzyme B expression in total CD8 + T cells from the BAL was determined by flow cytometry. Solid histogram represents fluorescence minus one control. Data are pooled from two experiments $(n=4-6)$. Granzyme B expression in tetramer-specific CD8+ T cells from the BAL was determined by flow cytometry. Data pooled from two experiments $(n=3-6)$. Error bars represent s.e.m. MFI, mean fluorescence intensity. 
restimulation (Figure 1e) and decreased Granzyme B expression by both total (Figure 1f) and NP 366-374 tetramer-binding CD8 + $\mathrm{T}$ cells (Figure 1f). Taken together, these data demonstrate that TSLPR signaling promotes influenza-specific CD8 + T-cell responses.

TSLP does not regulate the survival of lymphocytes recruited to the site of infection

TSLP has been previously linked to promoting CD8+ T-cell survival in vivo and in vitro. ${ }^{16}$ To address whether TSLP directly enhances the survival of lung-recruited lymphocytes, we measured apoptosis and death of total CD8 + and influenza-specific CD8 + T cells in the bronchoalveolar lavage of mice 10 days after infection (Figure 2a). We observed no significant differences in the rate of cell death or apoptosis of these cell populations. Furthermore, TSLPR-deficient influenza-specific CD8 + T cells expressed significantly higher levels of the antiapoptotic molecule Bcl-2 (B-cell lymphoma 2; Figure 2b), which could be indicative of a less mature activation state. ${ }^{22}$ a
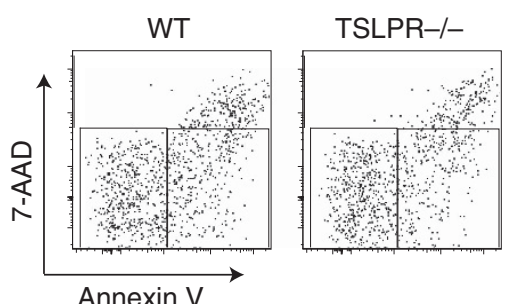

Gated on NP+ CD8+ T cells in BAL

Dead Apoptotic

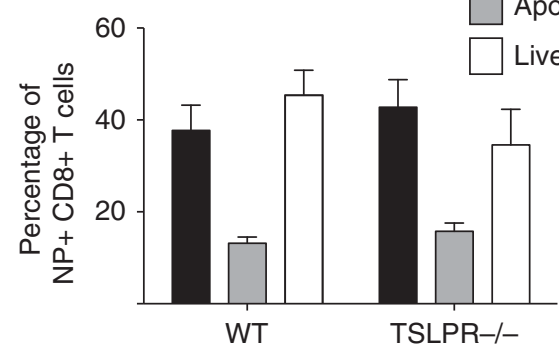

Gated on all CD8+ T cells in BAL
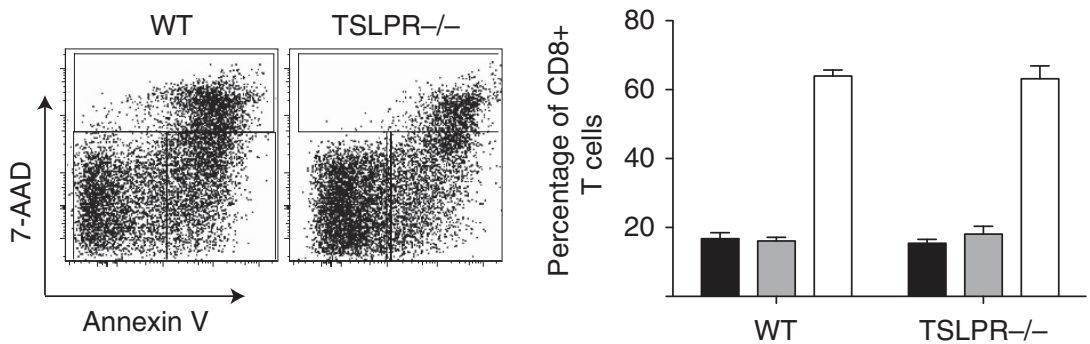

b

$\mathrm{NP}+\mathrm{CD} 8+\mathrm{T}$ cells in BAL
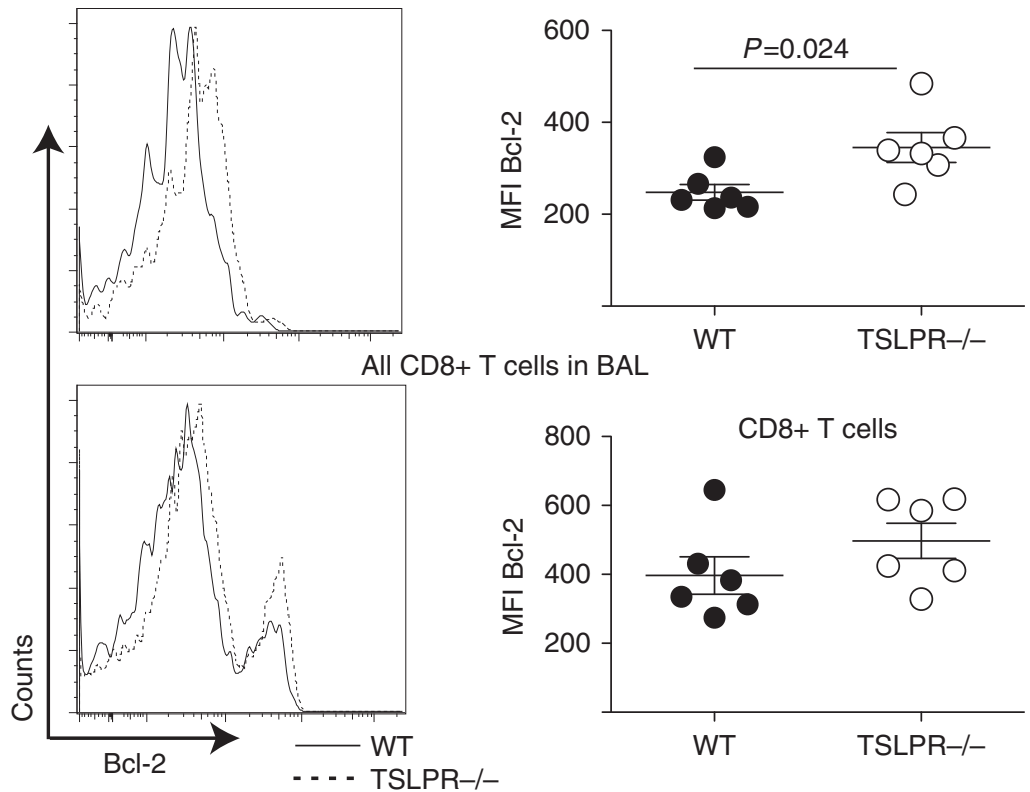

Figure 2 Thymic stromal lymphopoietin (TSLP) does not regulate survival of lymphocytes recruited to the site of infection. Wild-type (WT) and TSLP receptor (TSLPR)-deficient mice were infected intranasally with 50 PFU PR8 influenza virus. (a) On day 10 after infection, mice were killed and the indicated cell populations infiltrating the bronchoalveolar lavage (BAL) were analyzed for cell death (7-AAD+, Annexin $V+)$ and apoptosis (7-AAD-, Annexin $\mathrm{V}+$ ) by flow cytometry. 7-AAD, 7-aminoactinomycin D. (b) Expression of the antiapoptotic molecule Bcl-2 (B-cell lymphoma 2) was assessed in the indicated cell populations by flow cytometry. Data are representative of at least three independent experiments $(n=4-5)$. 
TSLP acts indirectly to augment influenza-specific CD8 + T-cell responses

Various hematopoietic cells including DCs and CD8 + and $\mathrm{CD} 4+\mathrm{T}$ cells express the TSLPR. Hence, TSLP could act directly and/or indirectly on CD8 $+\mathrm{T}$ cells to promote their activation. To distinguish the primary target cell of TSLP during influenza infection, we generated mixed bone marrow chimeras by reconstituting lethally irradiated wild-type hosts with a mixture of wild-type congenic (CD45.1) and TSLPR-deficient (CD45.2) donor bone marrow in a 1:1 ratio. At 2 months after reconstitution, chimeric mice were infected with influenza virus and the immune response in the wild-type or knockout compartment was assessed. Wild-type and TSLPR-deficient T cells exhibited comparable influenza-specific responses as measured by the frequency of tetramer-positive CD $8+\mathrm{T}$ cells (Figure 3a), cytokine production (Figure $3 \mathbf{b}$ ), and Granzyme B expression (Figure 3c,d). Moreover, TSLPR-deficient CD8 + $\mathrm{T}$ cells recruited to the airways following infection did not exhibit compromised survival (Figure 3e). These data demonstrate that TSLP acts indirectly, possibly via antigenpresenting cells, to boost influenza-specific CD $8+\mathrm{T}$-cell responses.
TSLP does not affect antigen uptake, transport, or priming of influenza-specific responses in the draining lymph node

We investigated if the absence of TSLP signaling could influence the uptake of viral antigen by DCs and their subsequent migration and presentation to T cells in the draining lymph node. Wild-type and TSLPR-deficient mice were infected with influenza virus in the presence of fluorescein isothiocyanate (FITC)-labeled ovalbumin (OVA) and the ensuing migration of airway DCs to the draining mediastinal lymph node was monitored. TSLPR-deficient DCs showed no impairment in antigen uptake or migration into the draining lymph node (Figure 4a). To assess the capacity of the migrated DCs to activate specific $T$ cells, we infected wild-type and TSLPR-deficient mice with influenza A (PR8-SIINFEKL) that expresses the MHC-I (major histocompatibility complex class I)-restricted OVA peptide (amino-acid sequence SIINFEKL). ${ }^{23}$ DCs isolated from the lymph nodes of infected TSLPR-deficient mice were equally efficient in inducing proliferation of OVA-peptide-specific TCR-transgenic OT-I CD $8+\mathrm{T}$ cells ${ }^{24}$ in vitro (Figure 4b), indicating that TSLPR signaling was not required for antigen presentation by DCs in the draining lymph node. We also characterized the DC subsets in the draining lymph node and observed similar kinetics and expression of costimulatory a

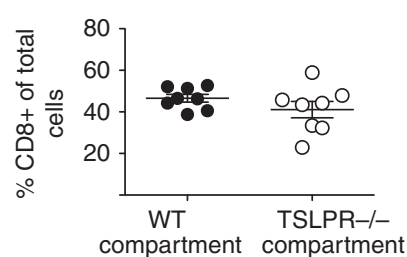

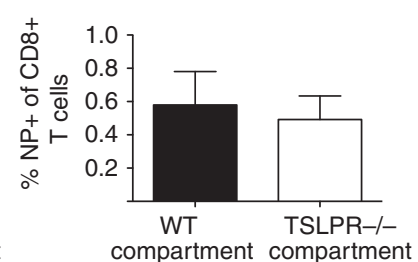

b

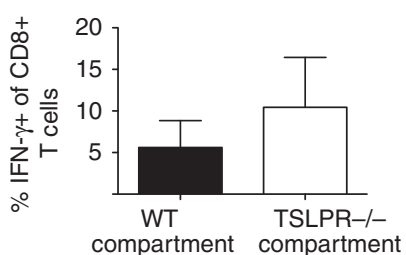

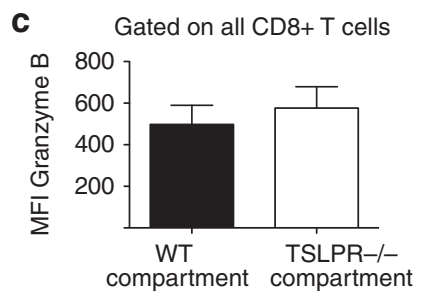
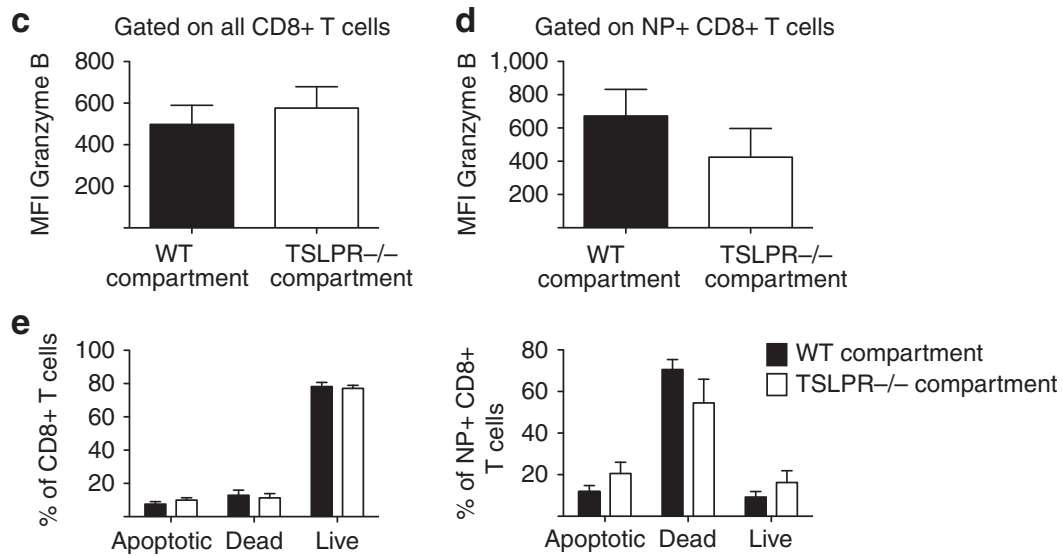

Figure 3 Thymic stromal lymphopoietin (TSLP) acts indirectly to augment influenza-specific CD8 + T-cell responses. Lethally irradiated wild-type mice (WT) were reconstituted with bone marrow from WT (Ly5.1) and TSLP receptor (TSLPR)-deficient (Ly5.2) mice in a 1:1 ratio. At 8 weeks after reconstitution, chimeric mice were infected with 50 PFU PR8 and influenza-specific responses in the bronchoalveolar lavage (BAL) were monitored on day 10 after infection. (a) Percentage of CD8 + T cells from the WT or TSLPR-deficient compartment was analyzed by flow cytometry. The percentage of NP 366-374 tetramer + cells within CD8 + T cells of either the WT or TSLPR-deficient compartment was evaluated. (b) Viability of the indicated cells within the WT or TSLPR-deficient compartment was determined by using Annexin V and 7-aminoactinomycin D (7-AAD). Granzyme B expression in (c) total and (d) NP 366-374 tetramer + CD8+ T cells within the WT or TSLPRdeficient compartment was measured. MFI, mean fluorescence intensity. (e) Cells from the BAL of infected mice were restimulated with bone marrow-derived dendritic cells (BMDCs) pulsed with influenza virus for $6 \mathrm{~h}$ in the presence of monensin and the proportion of interferon- $\gamma$ (IFN- $\gamma$ )-producing cells within the WT or the TSLPR-deficient CD8 + T-cell compartment was determined. Data are pooled from two independent experiments $(n=3-5)$. 
a
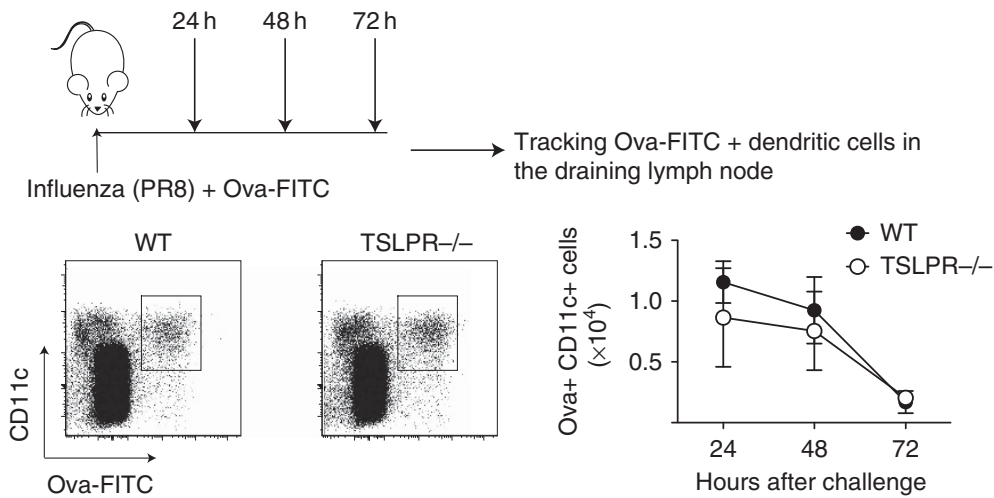

Hours after challenge

b

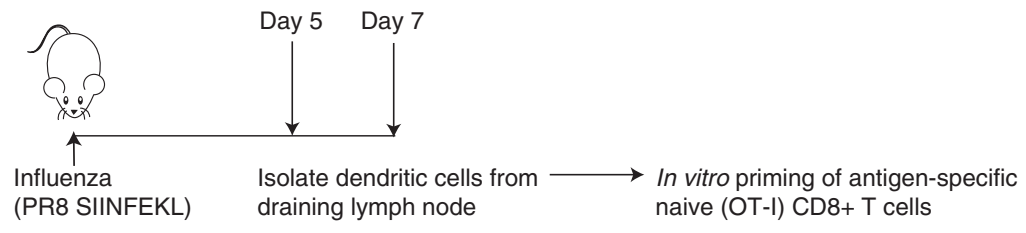

Dendritic cells from the draining lymph node
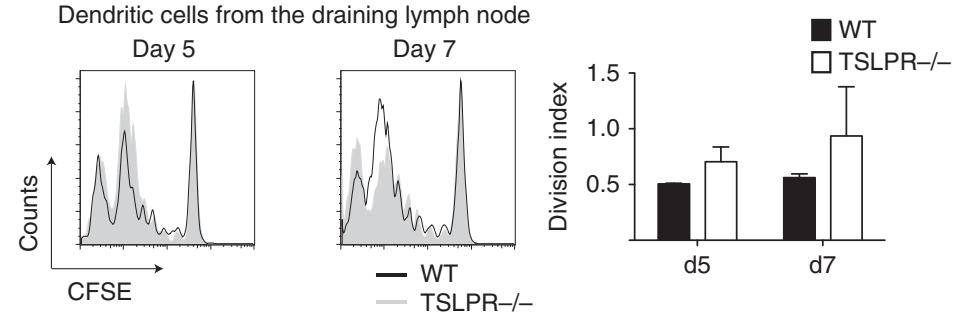

C
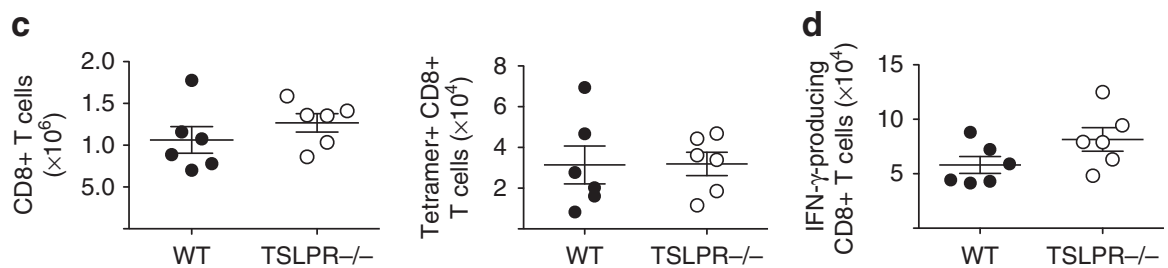

Figure 4 Thymic stromal lymphopoietin (TSLP) does not affect priming of T-cell responses in the draining lymph node but affects DC function in the lung. Wild-type (WT) and TSLP receptor (TSLPR)-deficient mice were administered $50 \mu \mathrm{g}$ Ova-FITC (ovalbumin-fluorescein isothiocyanate) together with 50PFU PR8 intranasally. (a) Migration of airway dendritic cells (DCs) transporting Ova-FITC to the lung draining lymph node was monitored by flow cytometry at the indicated time points after challenge. Data are representative of two independent experiments $(n=3)$. (b) WT and TSLPRdeficient mice were infected intranasally with 50 PFU recombinant PR8-SIINFEKL influenza virus. As indicated, either on day 5 or day 7 after infection, $\mathrm{CD} 11 \mathrm{c}+$ cells were sorted by magnetic bead separation from the draining lymph nodes of infected mice and cultured together with carboxyfluorescein succinimidyl (CFSE)-labeled OT-I transgenic CD8 + T cells for $72 \mathrm{~h}$. Black line represents WT mice and solid histogram represents TSLPR-deficient mice. Division index was determined using FlowJo software and represents the average number of divisions a cell has undergone. WT and TSLPRdeficient mice were infected with 50 PFU PR8 and (c) number of total CD8 + T cells and influenza-specific NP 366-374 tetramer+ CD8+ T cells in the mediastinal lymph node was assessed on day 10 after infection by flow cytometry. Data are representative of at least three independent experiments $(n=3-6)$. (d) Mediastinal lymph node cells were isolated from infected mice day 10 after infection and restimulated in vitro by bone marrow-derived dendritic cells (BMDCs) pulsed with ultraviolet (UV)-inactivated virus for $6 \mathrm{~h}$ before analysis of interferon- $\gamma$ (IFN- $\gamma$ ) production. Data are representative of two independent experiments $(n=5-6)$.

molecules (Supplementary Figure S3 online). In further support of these data, we found similar frequencies of virus-specific $\mathrm{CD} 8+\mathrm{T}$ cells in the lung-draining lymph nodes (Figure 4c), which exhibited comparable capacity to produce proinflammatory cytokines (Figure 4d).

\section{TSLP acts on DCs and consequently affects local reactivation of $T$ cells in the lungs and airways}

Based on our earlier data we hypothesized that TSLP might rather influence local responses at the site of infection. DCs present in the lung are critical for the reactivation of effector and memory T cells and the consequent clearance of the virus. ${ }^{6}$ Furthermore, they are known to express TSLPR and respond to TSLP in vitro. ${ }^{15,17,25}$ Thus, we investigated DC function at the site of infection. We found that in contrast to the mediastinal lymph node (Figure 4b), DCs purified from the lungs of influenza (PR8-SIINFEKL)-infected TSLPR-deficient mice were compromised in their ability to induce proliferation of antigen-specific OT-I CD8 + T cells (Figure 5a). In addition, we also observed a similar defect in the TSLPR-deficient DCs 
a

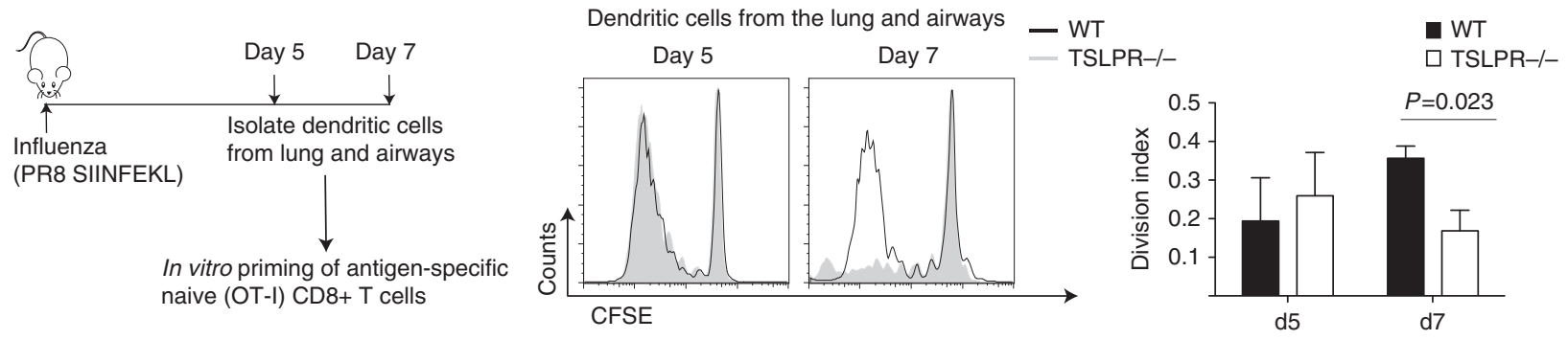

b

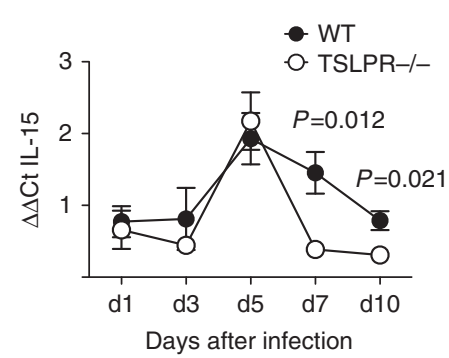

c

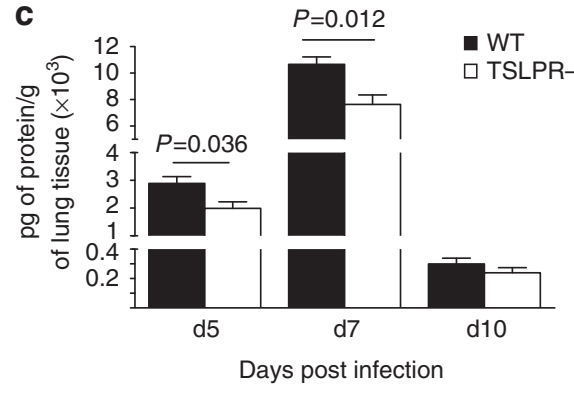

d

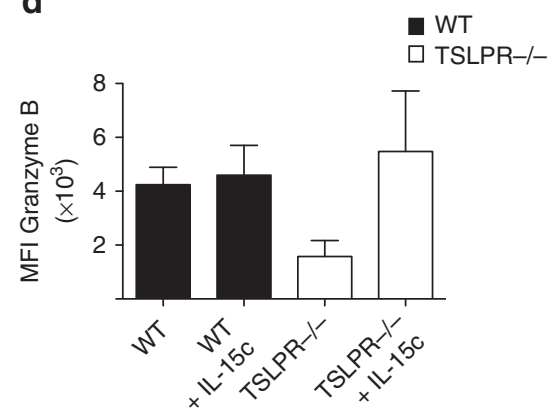

e

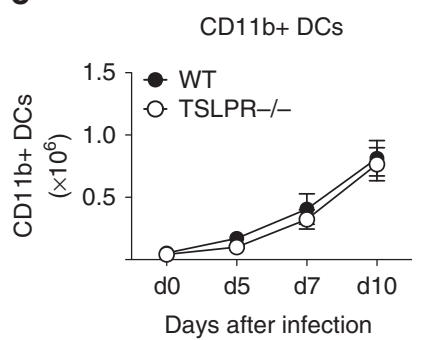

f

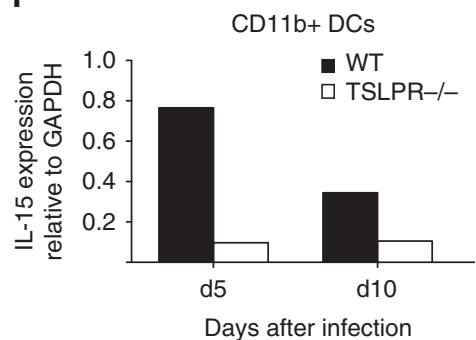

g

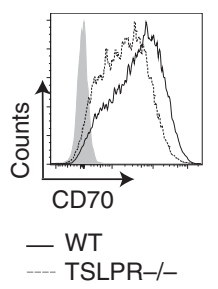

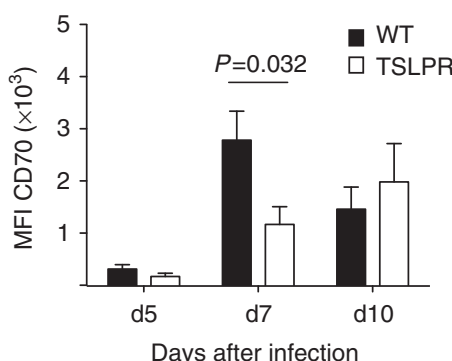

Figure 5 Thymic stromal lymphopoietin (TSLP) regulates interleukin-15 (IL-15) trans-presentation by CD11b+ inflammatory dendritic cells (DCs). (a) Wild-type (WT) and TSLP receptor (TSLPR)-deficient mice were infected intranasally with 50 PFU recombinant PR8-SIINFEKL influenza virus. On day 5 or day 7 after infection, CD11c + cells were sorted by magnetic bead separation from the lungs of infected mice and cultured together with carboxyfluorescein succinimidyl (CFSE)-labeled OT-I transgenic CD8 + T cells for $72 \mathrm{~h}$. Division index was determined using FlowJo software and represents the average number of divisions a cell has undergone. Black line represents WT mice and solid histogram represents TSLPR-deficient mice. (b) IL-15 expression was determined by quantitative reverse-transcriptase-PCR (RT-PCR). Results were first normalized to glyceraldehyde-3phosphate dehydrogenase (GAPDH) expression, and subsequently to expression by naive lungs to calculate the $\Delta \Delta \mathrm{Ct}$. Data were pooled from two independent experiments $(n=3-5)$. (c) IL-15 and IL-15/IL-15R $\alpha$ protein levels were determined in lung homogenate from infected mice at indicated time points after infection $(n=4-5)$. (d) Mice were infected with 50PFU PR8 and on day 9 after infection given either phosphate-buffered saline (PBS) or IL15/IL15R complexes (IL-15c) intranasally. Granzyme B expression by influenza-specific CD8 + T cells in the airways was assessed 12 $\mathrm{h}$ later. $\mathrm{MFI}$, mean fluorescence intensity. (e) Lung-recruited inflammatory CD11b + DCs were quantified by flow cytometry at the indicated time points after infection. (f) Mice were infected with 50PFU PR8 and CD11b + DCs were sorted by flow cytometry from pooled lungs $(n=3-4)$ of either WT or TSLPRdeficient mice at indicated time points after infection. The IL-15 mRNA level was measured in these DCs. (g) CD70 expression was determined on the $\mathrm{CD} 11 \mathrm{~b}+$ inflammatory DC subset on indicated days after infection. Data are representative of at least two independent experiments $(n=3-5)$.

to prime effector $\mathrm{CD} 8+\mathrm{T}$ cells isolated from the lung of mice infected with influenza A (Supplementary Figure S6 online). As DCs shape the inflammatory milieu at the site of infection, we additionally compared the expression of cytokines that are crucial for efficient CD8 $+\mathrm{T}$ cells responses in the lungs of mice. Among these, we found that the expression of IL-15 was reduced in the TSLPR-deficient mice (Figure 5b,c). Furthermore, recombinant TSLP enhanced IL-15 expression in bone marrow-derived DCs cultured with ultraviolet-irradiated PR8 (Supplementary Figure S1 online). Reconstitution of TSLPR-deficient mice with IL-15/15R complex partially restored the activation of CD8 + T-cell response in the lung as character- ized by Granzyme B expression in influenza-specific CD8 + T cells (Figure 5d). In particular, the trans-presentation of IL-15 by DCs at the site of infection has been shown to regulate local virus-specific responses. ${ }^{26}$ We tracked different DC subsets and their activation states in lungs of infected mice during the course of infection (Figure 5e, Supplementary Figures S2, S4, and $\mathbf{S 5}$ online). Of note was the CD11b + DC subset, which expressed the highest levels of TSLPR (Supplementary Figure S4 online). Although the recruitment of these cells was similar in both wild-type and TSLPR-deficient mice (Figure 5e), their ability to produce IL-15 was reduced in the absence of TSLPR (Figure 5f, Supplementary Figure S5 online). This could in 
part explain the defective pulmonary CD8 + T-cell responses as DC-derived IL-15 is important for the local restimulation of $\mathrm{CD} 8+\mathrm{T}$ effector cells. ${ }^{26}$

We also characterized the expression of several costimulatory molecules including CD40, CD80, CD86, and CD70, which are involved in DC/CD8 + T-cell interactions. We found that TSLP specifically influenced the expression of the costimulatory molecule CD70. It has been shown that CD70 expression by DCs is important for the generation of influenza-specific CD8 + T-cell responses. ${ }^{27}$

We observed that the expression of CD70 by the CD11b + DCs at day 7 after infection was decreased in the absence of TSLPR (Figure 5g). Interestingly, TSLP also affected CD70 expression by the CD11b - CD103 + DC subset (Supplementary Figure S4B online).

Taken together, our data indicate that TSLP primarily acts to enhance maturation of newly recruited CD11b + inflammatory DCs by engaging specific pathways, including the production of IL-15 and the expression of costimulatory molecule CD70. The inflammatory DCs, matured in the presence of TSLP during viral infection, are more potent at restimulation of local CD8 + T-cell responses in the lung (Figure 6).

\section{DISCUSSION}

Although TSLP has been shown to directly regulate CD8 + $\mathrm{T}$-cell homeostasis, our data from mixed bone marrow chimeras indicated that during viral infection TSLP affected CD8 + $\mathrm{T}$ cells indirectly by signaling on another hematopoietic cell type (Figure 3). TSLP is known to regulate DC maturation, and our data indicate that it acts locally in the lung to regulate the function of newly recruited inflammatory DCs. In line with our work, studies utilizing human cells have linked TSLP as an adjuvant in BCG (Bacillus Calmette-Guérin)-mediated antitumor cytotoxic CD8 + T-cell responses ${ }^{18}$ by signaling on DCs, although the underlying molecular mechanism of this activity remains unknown.

DCs are constantly sampling the airways and, on infection, transport viral antigen to the draining lymph nodes where they initiate protective CD8 + T-cell responses. Concurrently, DC precursors are recruited to the infected lungs, where they differentiate locally into various DC subsets. In the lymph nodes, both migratory CD11b - and CD11b + DCs are potent at cross-presenting viral antigen. ${ }^{3,27}$ The predominant role for one subset over the other is attributed to a difference in infectious dose. It is speculated that at a lower dose of infection, CD11b + DCs infected by the virus do not undergo apoptosis and are hence able to migrate and cross-present the antigen directly, ${ }^{27,28}$ and at higher doses of infections, because of an increase in virus-induced cell death, the CD11b - DCs crosspresent antigen more owing to their ability to phagocytose apoptotic cells. $3,27,28$

In our study, which also uses a low dose of infection, the initiation of influenza-specific CD8 + T-cell responses in the lymph node was independent of TSLP. The different DC subsets migrated to the draining lymph nodes with similar kinetics and expressed similar if not even higher levels of costimulatory molecules in the TSLPR-deficient mice (Figure 4, Supplementary Figure S3 online). Consequently, the influenza-specific T-cell responses in the lymph node were unaltered. We also observed that CD11b + DCs provided costimulation via CD70 in the lymph nodes, ${ }^{27}$ but this was independent of TSLP (Supplementary Figure S3 online). TSLP expression peaked at day 5 after infection, coinciding with the peak of the viral replication and the transition from the innate to the adaptive immune response in the lung (Figure 1a).

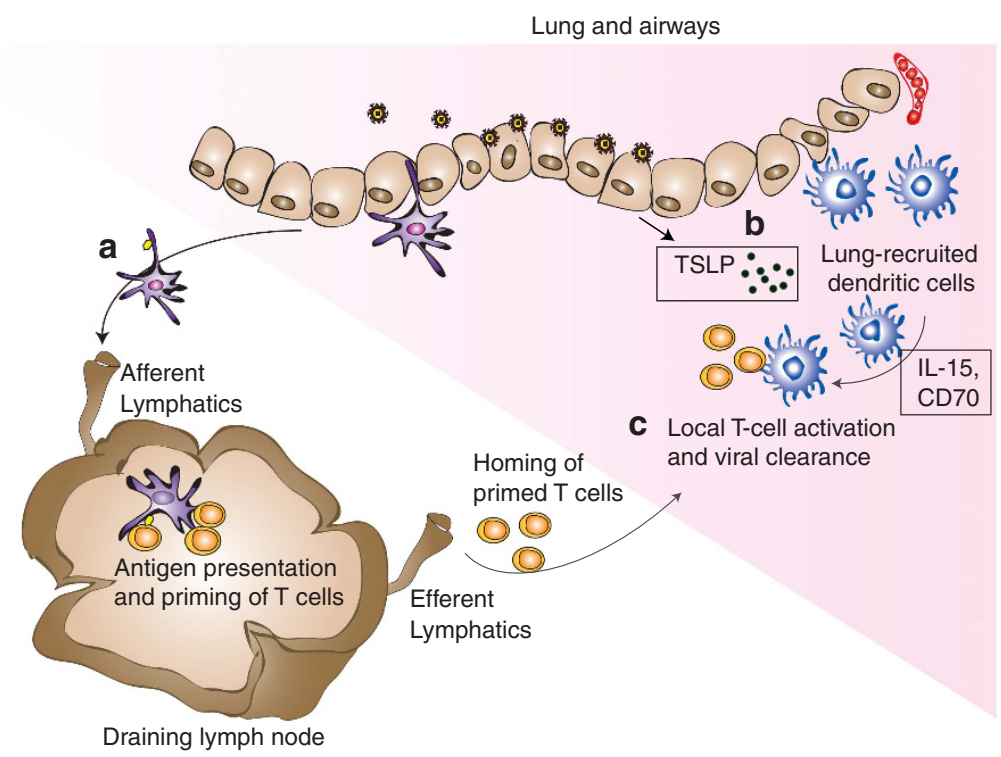

Figure 6 Thymic stromal lymphopoietin (TSLP) enhances pulmonary influenza-specific CD8+ T-cell responses. (a) TSLP does not affect the initiation of adaptive immune response in the draining lymph node. (b) It acts on CD11b+inflammatory DCs recruited to the lung and (c) induces the production of interleukin-15 (IL-15) and expression of CD70, which enhances influenza-specific CD8+ T-cell responses. 
It is likely that the maturation of the DCs that migrate to the lymph nodes is dependent on factors preceding TSLP expression in the lung. Thus, the reduced pulmonary influenza-specific CD8 + T-cell response in the TSLPR-deficient mice resulted from the absence of signaling of TSLP on cells within the lung.

The effector CD8 + T cells, which are recruited to the lungs, require an additional interaction with the DCs in the lung. ${ }^{6,7,29}$ Indeed, TSLPR-deficient DCs isolated at day 7 from infected lungs showed an impaired ability to prime antigen-specific T-cell responses (Figure 5a, Supplementary Figure 6 online). As different DC subsets have been attributed specific functions during infection, ${ }^{28}$ we investigated whether TSLP targets a specific DC subset in the lungs. Monocyte-derived CD11b + DCs are the predominant population when the first effector CD8 + $\mathrm{T}$ cells get recruited to the lungs. ${ }^{1,2} \mathrm{~A}$ subpopulation of the CD11b + DCs, the tip DCs, are also proposed to support the local proliferation of effector CD8 + T cells in the lungs. ${ }^{30} \mathrm{In}$ our study the CD11b + DC subset expressed the highest level of the TSLPR and was thus likely to be more responsive to cues from TSLP (Supplementary Figure S4A online). In line with this, TSLP specifically targeted the function of these DCs by influencing expression of the costimulatory molecule CD70 as well as the production of IL-15 (Figure 5f,, $\mathbf{g}$ ). Costimulation via $\mathrm{CD} 70$ on $\mathrm{CD} 11 \mathrm{~b}+\mathrm{DCs}$ licenses them to expand CD8 + T cells in the lymph node, ${ }^{27}$ but their function in the lung has not been addressed before. We observed that TSLP affected the expression of CD70 by inflammatory CD11b + DCs in the lungs (Figure 5g). We also saw a similar defect in the CD11bDCs in the lung, although they expressed much lower levels of CD70 in comparison with the CD11b + DCs (Supplementary Figure S4B online). The expression of other costimulatory molecules such as CD40, CD80, and CD86 by various DC subsets in the lungs was unaffected in the absence of TSLPR signaling (Supplementary Figure S4 online). As in the lymph nodes, ${ }^{27}$ $\mathrm{CD} 8+\mathrm{T}$ effector cells recruited to the lungs might also exhibit a differential requirement for costimulatory molecules and possibly a greater dependence on costimulation via CD70.

Another pathway affected by the absence of TSLPR signaling on the CD11b + DCs was the production of IL-15. Trans-presentation of IL- 15 by pulmonary DCs has been shown to regulate CD8 + T-cell survival within the lung following influenza infection. ${ }^{26}$ However, this study focused on early time points and not at the peak of the effector response. In other settings of inflammation, IL-15 has been reported to activate antigenspecific CD8 + T cells. ${ }^{31,32}$

We observed that TSLPR signaling acted to directly promote IL-15 expression in DCs in vitro (Supplementary Figure S1 online). We then followed the kinetics of IL-15 production during the course of influenza infection and found that the production of IL-15 is compromised in the absence of TSLPR signaling (Figure 5c).

Interestingly, although we were able to detect differences in mRNA expression of IL-15 from total lung cells only at day 7 and day 10, we observed differences in the protein levels (IL-15 and IL-15/IL-15R $\alpha$ complexes) as early as day 5 after infection. To consider whether temporal differences in the source of IL-15 in the lungs accounted for this inconsistency, we purified different cells from infected lungs and looked at the expression of IL-15 mRNA. We were able to detect differences in the expression of IL-15 in the CD11b + DCs even at day 5 after infection (Supplementary Figure S5A online). At this time, we observed that sorted cell fractions containing macrophages and plasmacytoid DCs also expressed IL-15 but this was independent of TSLPR deficiency (Supplementary Figure S5A online). As a decreased expression of IL-15 mRNA by CD11b + DCs correlated with the decreased protein levels, this suggested that the CD11b + DCs were the chief producers of IL-15. In further support, the expression of IL-15R $\alpha$, which is believed to faithfully represent the production of IL- $15,{ }^{26}$ is also highest on the CD11b + DCs (Supplementary Figure S5B online).

Both CD70 and IL-15 are crucial for influenza-specific CD8 + T-cell responses, although via different pathways. ${ }^{26,27}$ Engagement of the CD70 ligand ${ }^{27}$ on $\mathrm{CD} 8+\mathrm{T}$ cells has been shown to aid in proliferation, whereas IL-15 has been shown to influence the survival ${ }^{26}$ of effector CD8 $+\mathrm{T}$ cells recruited to the lung. We partially restored the CD8 + T-cell function by administering IL-15 complexes in the TSLPR-deficient mice. This suggested that these pathways are regulated by TSLP independently of each other. Furthermore, TSLP enhanced the expression of CD70 on both CD11b - and CD11b + DCs in the lung, whereas it specifically boosted IL-15 expression by the CD11b + DCs.

In conclusion, we show a novel role for TSLP in engaging distinct activation pathways of CD11b + inflammatory DCs during influenza infection and consequently augmenting antiviral CD8 + T-cell responses.

\section{METHODS}

Mice and infections. C57Bl/6 mice aged between 8 and 14 weeks were purchased from Charles River (l'Arbresle Cedex, France), whereas transgenic OT-I mice and TSLPR-deficient mice were bred locally. Influenza virus strain PR8 (A/Puerto Rico8/34, H1N1) was sourced from Virpur (Virapur LLC, San Diego, CA). Viral infections were performed by intranasal administration of $50 \mathrm{PFU}$ of virus in $50 \mu \mathrm{l}$ of phosphate-buffered saline. Animal experiments were performed in accordance with the institutional guidelines and Swiss federal and cantonal laws on animal protection. The reverse engineered influenza PR8 SIINFEKL strain ${ }^{23}$ was kindly provided by Dr Richard Webby (St Jude Children's Research Hospital, Memphis, TN).

TCID 50 assay. Influenza virus was quantified from lung homogenate using the TCID 50 assay. ${ }^{33}$ MDCK (Madin-Darby canine kidney) cells were infected overnight with tissue homogenate and incubated for 5 days at $37^{\circ} \mathrm{C}$. At the end of 5 days, infectious virus was quantified by adding gluteraldehyde-fixed guinea pig red blood cells and TCID 50 was calculated for each sample by reading the virus-induced agglutination of red blood cells.

In vivo DC tracking. Wild-type and TSLPR-deficient mice were administered $50 \mu \mathrm{g}$ Ova-FITC (Invitrogen, Grand Island, NY) together with 50 PFU PR8 intranasally. Migration of airway DCs transporting Ova-FITC to the lung-draining lymph node was monitored by flow cytometry.

Antibodies and flow cytometry. Different combinations of the following antibodies were used for flow cytometry. For analysis of DC subsets in the lung a combination of CD11b PerCP-Cy5.5, CD11c APC-Cy7, B220 FITC, Ly6c pacific blue, NK1.1 PE-Cy7, MHC II Alexa Fluor 700, PDCA1 Alexa Fluor 647, CD103 PerCP-Cy5.5, CD40 PE, CD70 PE, CD80 PE, or 
CD86 PE was used. TSLPR and IL-15R $\alpha$ antibodies were purchased from R\&D (Minneapolis, MN). Influenza-specific CD8 + T cells were stained with NP tetramer 366-374, PA, and PB1 generated in-house. For detecting cell death and apoptosis, a combination of Annexin $\mathrm{V}$ and 7-AAD (7-aminoactinomycin D) was used. Intracellular staining was performed to detect the expression of Bcl-2, interferon- $\gamma$, and Granzyme B.

All antibodies were purchased from Biolegend (San Diego, CA) unless indicated. Stained cells were acquired on a BD FACS Calibur or BD FACS CANTO or BD FACS LSR II (Franklin Lakes, NJ) and analyzed on FlowJo software (Tree Star, Ashland, OR).

Ex vivo restimulation of specific CD8 + T cells. Bone marrow-derived DCs were pulsed with ultraviolet-irradiated influenza virus overnight. The following day, cells from the bronchoalveolar lavage of infected mice were cocultured with them for $6 \mathrm{~h}$ in the presence of monensin and assessed for the production of interferon- $\gamma$ by flow cytometry.

In vitro coculture of DCs and CD8 + T cells. DCs were isolated by positive selection with CD11c+ beads (Miltenyi Biotech, Bergisch Gladbach, Germany) from infected lungs and put in culture with either MACS-sorted naive splenic CD8 + T cells or effector CD8 + T cells from infected lungs in a ratio of 1:5. Proliferation was determined by dilution of carboxyfluorescein succinimidyl ester-labeled CD8 + T cells.

In vivo reconstitution of IL-15. IL-15 complexes were generated as described previously. ${ }^{34} \mathrm{~A}$ total volume of $1.5 \mu \mathrm{g}$ of IL- 15 complexed with $7 \mu \mathrm{g}$ of IL-15R $\alpha$ was administered intranasally in a volume of $78 \mu \mathrm{l}$ per mouse at day 9 after infection. The analysis was performed $24 \mathrm{~h}$ after administration of the IL-15 complex.

Enzyme-linked immunosorbent assay. IL-15 levels in lung homogenate of infected mice were determined using IL-15 ELISA DuoSet from R\&D systems.

Generation of mixed bone marrow chimeras. Lethally irradiated wildtype mice were reconstituted with bone marrow from wild-type (Ly5.1) and TSLPR-deficient (Ly5.2) mice in a 1:1 ratio. Chimeric mice were infected 8 weeks after reconstitution.

Quantitative real-time PCR. Total RNA was purified from cells obtained from whole lung and trachea of mice using Tri reagent. Real-time PCR was carried out according to the manufacturer's instructions using the quantifast SYBR green RT-PCR kit (Qiagen, West Sussex, UK). The following primers were used: IL-15 forward, 5' -CATCCATCTCGTGCTACTTGTG TT-3'; IL-15 reverse, 5' -CATCTATCCAGTTGGCCTCTGTTT-3'; TSLP forward $5^{\prime}$-AGAGAAGCCCTCAATGACCA-3'; TSLP reverse 5'-TCTGGAGATTGCATGAAGGA-3'; GAPDH forward 5' -GGGTGT GAACCACGAGAAAT- ${ }^{\prime}$; GAPDH reverse 5' -CCTTCCACAATGC CAAAGTT-3'; Influenza matrix protein forward 5' -GGACTGCAG CGTAGACGCTT- ${ }^{\prime}$, reverse $5^{\prime}$-CATCCTGTTGTATATGAGGCCC AT- $3^{\prime} ; \beta$ actin forward $5^{\prime}$-CCCTGAAGTACCCCATTGAAC- $3^{\prime}$; $\beta$ actin reverse $5^{\prime}$-CTTTTCACGGTTGGCCTTAG-3'. Expression was determined either by using absolute quantification or by comparative $\Delta$-threshold cycle method using GAPDH as a comparator. IL-15 and TSLP expression is represented as fold change relative to naive lung sample.

Flow cytometric sorting of DC subsets. Wild-type and TSLPR-deficient mice were infected with 50 PFU PR8 intranasally. Lungs from 3 to 4 mice were pooled and stained for sorting by flow cytometry. DC subsets were gated as described (Supplementary Figure S5 online) and sorted into Trizol (Invitrogen). RNA was isolated from the different sorted populations and real-time PCR was carried out as described above.

Statistical analysis. Student's $t$-test (unpaired, two tailed) was used to calculate significance levels between treatment groups. The $P$-values of $<0.05$ were considered significant. Graph generation and statistical analyses were performed using Prism version 5 software (GraphPad, La Jolla, CA). Standard error of the mean was used.

SUPPLEMENTARY MATERIAL is linked to the online version of the paper at http://www.nature.com/mi

\section{ACKNOWLEDGMENTS}

We thank Professor Daniel Speiser, Dr Christoph von Garnier, and Professor Hans Acha-Orbea for helpful discussions. The recombinant PR8-SIINFEKL was kindly provided by Professor Richard Webby. This project was funded by the Swiss National Science Foundation (310030.130029). B.J.M. is a Cloetta Medical Research Fellow.

\section{Author contributions}

K.Y., B.J.M. and N.L.H. designed research; K.Y. and A.S. performed research; I.F.L. contributed new reagents/analytic tools; K.Y. analyzed data; and K.Y., A.S., L.P.N., B.J.M., and N.L.H. wrote the paper.

\section{DISCLOSURE}

The authors declared no conflict of interest.

() 2013 Society for Mucosal Immunology

\section{REFERENCES}

1. GeurtsvanKessel, C.H. et al. Both conventional and interferon killer dendritic cells have antigen-presenting capacity during influenza virus infection. PLoS One 4, e7187 (2009).

2. GeurtsvanKessel, C.H. et al. Dendritic cells are crucial for maintenance of tertiary lymphoid structures in the lung of influenza virus-infected mice. J. Exp. Med. 206, 2339-2349 (2009).

3. GeurtsvanKessel, C.H. et al. Clearance of influenza virus from the lung depends on migratory langerin+CD11b - but not plasmacytoid dendritic cells. J. Exp. Med. 205, 1621-1634 (2008).

4. Topham, D.J. \& Doherty, P.C. Clearance of an influenza A virus by CD4+ T cells is inefficient in the absence of B cells. J. Virol. 72, 882-885 (1998).

5. Hufford, M.M., Kim, T.S., Sun, J. \& Braciale, T.J. Antiviral CD8+ T cell effector activities in situ are regulated by target cell type. J. Exp. Med. 208, 167-180 (2011).

6. McGill, J., Van Rooijen, N. \& Legge, K.L. Protective influenza-specific CD8 T cell responses require interactions with dendritic cells in the lungs. J. Exp. Med. 205, 1635-1646 (2008).

7. McGill, J. \& Legge, K.L. Cutting edge: contribution of lung-resident T cell proliferation to the overall magnitude of the antigen-specific CD8 $T$ cell response in the lungs following murine influenza virus infection. J. Immunol. 183, 4177-4181 (2009).

8. Plantinga, M., Hammad, H. \& Lambrecht, B.N. Origin and functional specializations of DC subsets in the lung. Eur. J. Immunol. 40, 2112-2118 (2010).

9. Ziegler, S.F. \& Artis, D. Sensing the outside world: TSLP regulates barrier immunity. Nat. Immunol. 11, 289-293 (2010).

10. Headley, M.B. et al. TSLP conditions the lung immune environment for the generation of pathogenic innate and antigen-specific adaptive immune responses. J. Immunol. 182, 1641-1647 (2009).

11. He, R. et al. TSLP acts on infiltrating effector T cells to drive allergic skin inflammation. Proc. Natl. Acad. Sci. USA 105, 11875-11880 (2008).

12. Zhou, B. et al. Reversal of thymic stromal lymphopoietin-induced airway inflammation through inhibition of Th2 responses. J. Immunol. 181, 6557-6562 (2008).

13. Massacand, J.C. et al. Helminth products bypass the need for TSLP in Th2 immune responses by directly modulating dendritic cell function. Proc. Natl. Acad. Sci. USA 106, 13968-13973 (2009).

14. Taylor, B.C. et al. TSLP regulates intestinal immunity and inflammation in mouse models of helminth infection and colitis. J. Exp. Med. 206, 655-667 (2009).

15. Ito, T. et al. TSLP-activated dendritic cells induce an inflammatory Thelper type 2 cell response through OX4O ligand. J. Exp. Med. 202, 1213-1223 (2005).

16. Rochman, Y. \& Leonard, W.J. The role of thymic stromal lymphopoietin in CD8+ T cell homeostasis. J. Immunol. 181, 7699-7705 (2008). 


\section{ARTICLES}

17. Gilliet, M. et al. Human dendritic cells activated by TSLP and CD4OL induce proallergic cytotoxic T cells. J. Exp. Med. 197, 1059-1063 (2003).

18. Sugimoto, H. et al. Thymic stromal lymphopoietin plays an adjuvant role in BCG-mediated CD8(+) cytotoxic T cell responses through dendritic cell activation. Clin. Immunol. (Orlando, FL) 136, 205-216 (2010).

19. Kinoshita, H. et al. Cytokine milieu modulates release of thymic stromal lymphopoietin from human keratinocytes stimulated with double-stranded RNA. J. Allergy Clin. Immunol. 123, 179-186 (2009).

20. Liu, Y.J. et al. TSLP: an epithelial cell cytokine that regulates T cell differentiation by conditioning dendritic cell maturation. Annu. Rev. Immunol. 25, 193-219 (2007).

21. Brandelius, A. et al. dsRNA-induced expression of thymic stromal lymphopoietin (TSLP) in asthmatic epithelial cells is inhibited by a small airway relaxant. Pulm. Pharmacol. Ther. 24, 59-66 (2011).

22. Grayson, J.M., Zajac, A.J., Altman, J.D. \& Ahmed, R. Cutting edge: increased expression of Bcl-2 in antigen-specific memory CD8+ T cells. J. Immunol. 164, 3950-3954 (2000).

23. Jenkins, M.R., Webby, R., Doherty, P.C. \& Turner, S.J. Addition of a prominent epitope affects influenza A virus-specific CD8+ T cell immunodominance hierarchies when antigen is limiting. J. Immunol. 177, 2917-2925 (2006)

24. Hogquist, K.A. et al. T cell receptor antagonist peptides induce positive selection. Cell 76, 17-27 (1994).

25. Hirano, R. et al. Human thymic stromal lymphopoietin enhances expression of CD80 in human CD14(+) monocytes/macrophages. Inflamm. Res. 60, 605-610 (2011).

26. McGill, J., Van Rooijen, N. \& Legge, K.L. IL-15 trans-presentation by pulmonary dendritic cells promotes effector CD8 T cell survival during influenza virus infection. J. Exp. Med. 207, 521-534 (2010).

27. Ballesteros-Tato, A., Leon, B., Lund, F.E. \& Randall, T.D. Temporal changes in dendritic cell subsets, cross-priming and costimulation via CD70 control CD8(+) T cell responses to influenza. Nat. Immunol. 11, 216-224 (2010).

28. Lambrecht, B.N. \& Hammad, H. Lung dendritic cells in respiratory viral infection and asthma: from protection to immunopathology. Annu. Rev. Immunol. 30, 243-270 (2012).

29. Legge, K.L. \& Braciale, T.J. Accelerated migration of respiratory dendritic cells to the regional lymph nodes is limited to the early phase of pulmonary infection. Immunity 18, 265-277 (2003).

30. Aldridge, J.R. Jr. et al. TNF/iNOS-producing dendritic cells are the necessary evil of lethal influenza virus infection. Proc. Natl. Acad. Sci. USA 106, 5306-5311 (2009).

31. Ma, L.L. et al. CD8 T cell-mediated killing of Cryptococcus neoformans requires granulysin and is dependent on CD4 T cells and IL-15. J. Immunol. 169, 5787-5795 (2002).

32. Rubinstein, M.P. et al. IL-7 and IL-15 differentially regulate CD8+ T-cell subsets during contraction of the immune response. Blood 112, 3704-3712 (2008).

33. Benton, K.A. et al. Heterosubtypic immunity to influenza A virus in mice lacking IgA, all Ig, NKT cells, or gamma delta T cells. J. Immunol. 166, 7437-7445 (2001).

34. Verbist, K.C., Cole, C.J., Field, M.B. \& Klonowski, K.D. A role for IL-15 in the migration of effector CD8 T cells to the lung airways following influenza infection. J. Immunol. 186, 174-182 (2010).

\section{(c)}

SOMERRIGHSRES

This work is licensed under the Creative Commons Attribution-NonCommercial-No Derivative Works 3.0

Unported License. To view a copy of this license, visit http://creativecommons.org/licenses/by-nc-nd/3.0/ 